\title{
Comparison of BMI and body fat percentages between national level teenage swimmers and controls
}

\begin{abstract}
Swimming is a popular sport in the world which competed at many different levels. Anthropometric parameters in teenagers including swimmers in Sri Lanka are not well documented. It is important to study on swimming and its effect on BMI and body fat levels because more children taking up swimming at school at competition levels.

The objective of the study was assessing the differences in anthropometric characteristics of teenage swimmers and non-swimmers. The specific objectives were to determine average BMI and body fat parameters of national level teenage swimmers and to compare with that of age and sex matched nonathletic and non-swimmers.

90 male and 90 female teenage swimmers who qualified for the School Nationals Meet were recruited to the study and also similar numbers of age, sex and geographical area matched controls. Standard equipment and procedures were used for measurements. Comparison was done using the independent sample $t$ test (SPSS 17.0).

In the results, statistical significance was not found in BMI scores and Lean Body Weight (LBW) of male swimmers $\left(M_{B M I}=22.8, S D=5.3\right.$ and $\left.M_{L B W}=52.3, S D=10.3\right)$ and non-swimmers $\left(M_{B M I}=23.7, S D=2.4\right.$ and $\left.M_{L B W}=48.7, S D=8.6\right)$ with $\mathrm{p}=0.15$ and 0.07 respectively while BMI and LBW of female swimmers $\left(M_{B M I}=19.0, S D=1.5\right.$ and $\left.M_{L B W}=42.0, S D=3.7\right)$ and nonswimmers $\left(M_{B M I}=22.3, S D=3.0\right.$ and $\left.M_{L B W}=39.3, S D=2.2\right)$ was statistically significant with a $\mathrm{p}$ value 0.0001 . Fat weight $(\mathrm{FW})$ and Fat percentage (Fat \%) of male swimmers $\left(M_{F W}=5.1\right.$, $S D=2.1$ and $\left.M_{F A T \%}=9.3, S D=4.6\right)$ and non-swimmers $\left(M_{F W}=7.7, S D=1.9\right.$ and $M_{F a^{\circ}=}=13.6$, $S D=3.9)$, and female swimmers $\left(M_{F W}=9.4, S D=1.9\right.$ and $\left.M_{F a 0_{\sigma}^{0}}=18.2, S D=3.4\right)$ and nonswimmers $\left(M_{F W}=13.3, S D=2.2\right.$ and $\left.M_{F a 0^{\circ}}=25.1, S D=3.1\right)$ were statistically significant with $\mathrm{p}$ values for FW 0.001 and 0.0001 and for Fat \% 0.001 and 0.0001 respectively.

Both male and female swimmers show a statistically significant difference in Fat weight and body fat percentages compare to the non swimmers. In height, weight, BMI and Lean body weight of the female swimmers show a statistical significance while male doesn't show in the comparison. Therefore swimming can be recommended to reduce the body fat which is a need of an individual's life from the childhood.
\end{abstract}

Keywords: anthropometry, BMI, body fat, teenage swimmers
Volume 4 Issue 6 - 2016

\author{
TDMSB Dassanayake,' Rajarathna AAJ,', \\ Rajarathna $S A^{2}$ \\ 'University of Colombo, Sri Lanka \\ 2Department of Physiology, University of Peradeniya, Sri Lanka
}

Correspondence: TDMSB Dassanayake, AUSU, Faculty of Medicine, University of Colombo, No 25 Kynsey Road, Colombo 08, Sri Lanka,Tel 00947I3009859, Email suranga@med.cmb.ac.lk

Received: December 14, 2016 | Published: May 31, 2016
Abbreviations: BMI, body mass index; FW, fat weight; Fat $\%$, fat percentage; LBW, lean body weight; SF, stroke frequency; SL, stroke length

\section{Introduction}

Swimming is a full body exercise that can be both fun and competitive. Competitive swimming ranges from school level to district, national and international championships, including the Olympic Games. With more and more children taking up swimming at school at competition levels, it has become important to know the effects of competitive swimming which include an intense regular training on their BMI and body fat levels. On the other hand the published data on the above mentioned anthropometric parameters, especially on swimmers are not readily available in Sri Lanka. Further the higher body fat levels and lower cardiorespiratory endurance the BMI values since child hood the increases the risk factors of noncommunicable disease. Lack of research and unavailability of data will affect the preventive measures nationally. Identification of methods such as involving in sports and further the best sport would benefit to fulfill the broad national objectives such as prevention of non-communicable diseases.

The aims of this study was to determine the mean BMI and body fat percentages of teen age (age 13-19years) swimmers and non swimmers in both genders, and to compare with the age and sex matched control groups.

Relationships between individual performances and body anthropometry were known for a long time and as a consequence a relationship has been established between body anthropometric characteristics and swimming. ${ }^{1}$ For the human body, anthropometric systems are mainly concerned with body build, composition and constitution and with the dimensions of the human beings. ${ }^{2}$

Anthropometry is used to assess and predict performance, health and survival of individuals ${ }^{3}$ and literature reveals that anthropometric variables have significant statistical correlation with performance components such as endurance. ${ }^{4}$ Also, different variables of 
anthropometry may be associated with performances ${ }^{5}$ and it further influences on specific sports which has a special relationship with the respective body parts.

Swimming performance is a multifactorial phenomenon of which the anthropometric aspects and energetic parameters play a significant role. ${ }^{6}$ Athletes who use high Stroke Frequency (SF), compared to the athletes who use high Stroke Length (SL), have a tendency to be wider in the sagittal and frontal planes of the middle part of the body, and less robust in the thorax. ${ }^{1}$ Generally the swimmers had higher muscle strength in the back and upper extremities.

Knechtle et al., ${ }^{5}$ showed that longer body segmental length contribute to successful swimming outcomes and the length of the upper arm related to race time, and pubescent boys presented a higher arm and thigh circumference in relation to none athletes boys, and this fact may be explained due to the training protocols influence on body anthropometry in teenage swimmers. ${ }^{8}$ This difference in the stature of swimmers may reflect the relationship between growth and development with the sport, swimming. ${ }^{8}$

Some particular research in specific sports such as swimming has found that there is no correlation between anthropometry and race performance. ${ }^{5}$ Generally physical conditioning of a swimmer including only body composition influences the performance. ${ }^{8}$ The authors ${ }^{8,9}$ have suggested for further studies on anthropometrical and muscular strength behavior in teenage population. Furthermore, swimming has been used by health professionals and exercise scientists as an exercise component of reducing body fat or body composition. ${ }^{10}$

\section{Materials and methods}

This descriptive analytical study evaluated anthropometrical measurements (height, body weight, BMI and body fat percentage) of teenage national level swimmers and the non-swimmer; non athlete teen ages in same age, sex and equal social levels. The general purpose of this study was to determine the above mentioned anthropometric values for teenage swimmers and non-athlete non swimmers and compare the groups.

The study population was the national level teen age swimmers who undergo an intense training as a routine.

The population for controls was the teenage individuals who have not participated in sports activities (Non-athletes/non swimmers) in interschool level (occasional athletes) or above or not participate in the sports activities at all.

The study was conducted based on the exercise physiology laboratory of Department of Physiology Faculty of Medicine, University of Peradeniya and the Allied Health Sciences Unit, Faculty of Medicine, University of Colombo. The assistance was obtained by the trained staff attached to the laboratories

The sample was extracted from the teenage swimmers (year 13 to 19 old) who qualified to swim for national champion ships and age group champion ships in Sri Lanka. The sample size was 90 for each group. The sample sizes were calculated using the formula

$$
n=\frac{2 \sigma^{2}}{\left(\mu_{1}-\mu_{2}\right)^{2}} \cdot f(\beta, \alpha)
$$

Where

$\sigma=$ highest standard deviation,

$$
\begin{aligned}
& \mu=\text { mean values of subjects and controls, } \\
& \sigma \text { fixed at } 95 \% ;
\end{aligned}
$$$$
\text { Relative precision } \rightarrow 20 \% \text { or less } \rightarrow \beta=20 \%
$$

The list of national swimming champion ship participants in 2011 was officially obtained from the Sri Lanka Aquatic Sports Union (SLASU) and created an excel sheet excluding the events based classification and the repeated names to create the individual swimmers list who qualified to swim at the national competitions. The teenage swimmers were extracted from the above list and two separate lists were made for males and females. The specific events (free style etc), distance (50m etc.) and age group (Under 14 etc.) specification was not considered in this study (Eg: Under 14 back stroke 50meters).

The selected pupils from the list by the names were identified and written informed consent obtained to participate in the study. Controls were selected to match age, sex and social status through the selected swimmers to the study. The consent was taken from the parents on the participant below 18years in all groups. The subjects who were recruited to the study was provided a pre test questionnaire and the successful candidates were provided information sheet and the consent forms to obtain the candidate's or the parent's consent.

Acute or chronic injuries and illness free subjects (age 13 -19) who had been involved swimming as a sport at least for 3 consecutive years to the recruitment date and had undergone more than 3 regular sessions out of 5 within the week prior to 2days of recruitment were included to the study. The attainment of puberty, the duration from the puberty on the date of recruitment and detailed reproductive aspects were not considered in the inclusion criteria. Ethics approval was obtained from Ethics Review committee of the Faculty of Medicine, University of Peradeniya.

The anthropometric measurements which were used to calculate the BMI were height and the weight of the subjects. The standard stedio meter ( $1 \mathrm{~mm}$ accuracy) and the weighing scale (in $\mathrm{Kg}$ with 2 decimal places accuracy) were used following the standard methods. Calibration of the equipment was carried out according to the necessity. The training for the swimmers was regular (Mornings 5.30-7.30) and the measurements were taken in between 10.30 to 11.30. Measurements of the controls were taken at the Allied Health Sciences Unit laboratory at the mentioned time slots on the days that the measurements were taken. The body fat percentage was calculated using the seven site skin folds method and the Jackson Pollok equation for men and women. All the measurements were taken 3times and the average value was taken for the data analysis. The hours and the days of the training were same for all age groups. BMI, lean body weight and the fat weight were calculated with simple mathematic equations using an excel data sheet. The data was initially recorded on printed sheets which were used for data collection and excel data base were maintained using standard recording protocols to collect, record and computerize the data.

The data was analyzed using SPSS version 17.0 (Statistical and Products Service Solutions, Chicago). The normality of data was checked using the QQ plot and the histogram. The means of data were compared in the swimmers and non-swimmers in male and female categories, using the independent sample $t$ test and the values were tabulated.

\section{Results}

Anthropometric measurements were obtained from 360 subjects, 
under four groups of equal size (90), namely male and female swimmers and male and female non-athletes, non-swimmers, between the ages of 13 and $19 \mathrm{yrs}$.

The individual data sets on body weight, height, shoulder breadth, hip circumference, lean body weight, fat weight, fat percentage and BMI had followed a normal distribution according to the histograms and QQ plots. The $\mathrm{p}$ value was considered less than or equal to 0.05 $(\mathrm{p} \leq 0.05)$ to be statistically significant in comparison using independent sample t-test under the condition of normal distribution of data.

The mean age, weight, height and BMI of male swimmers were $15 \pm 1.9,57.4 \pm 9.9 \mathrm{~kg}, 160 \pm 17.5 \mathrm{~cm}$ and $22.8 \pm 5.3 \mathrm{~kg} / \mathrm{m}^{2}$ respectively. The age and sex matched controls too had a mean age of $15 \pm 1.9$ years with mean weight $57.3 \pm 8.8 \mathrm{~kg}$ and mean height $156 \pm 12.8 \mathrm{~cm}$ and BMI the mean value $23.7 \pm 2.5 \mathrm{~kg} / \mathrm{m}^{2}$ (Table 1 ).

The mean age female swimmers were $15 \pm 1.8$ years and the mean body weight, height and BMI values were $51.4 \pm 4.3 \mathrm{~kg}, 164 \pm 5.5 \mathrm{~cm}$, and $19 \pm 1.5 \mathrm{~kg} / \mathrm{m}^{2}$ respectively. The age and sex matched female controls too had a mean age of $15 \pm 1.8$ years with the mean weight $52.6 \pm 3.7 \mathrm{~kg}$, mean height $153.0 \pm 8.3 \mathrm{~cm}$ and mean BMI $22.5 \pm 2.2 \mathrm{~kg} /$ $\mathrm{m}^{2}$ (Table 1).

Table I Comparison of mean values for weight, height, body mass index (BMI) of the groups $(P \leq 0.05)$

\begin{tabular}{llll}
\hline \multirow{2}{*}{ Group } & Mean & & \\
\cline { 2 - 4 } & Weight $(\mathbf{k g})$ & Height $(\mathrm{cm})$ & BMI $\left(\mathbf{k g} / \mathbf{m}^{2}\right)$ \\
\hline Male Swimmers & $57.4 \pm 9.9$ & $160.0 \pm 17.6$ & $22.8 \pm 5.3$ \\
Male Controls & $57.3 \pm 8.8$ & $156.0 \pm 12.8$ & $23.7 \pm 2.5$ \\
Female Swimmers & $51.4 \pm 4.3$ & $164.0 \pm 5.5$ & $19.0 \pm 1.5$ \\
Female Controls & $52.6 \pm 3.7$ & $153.0 \pm 8.3$ & $22.5 \pm 2.2$ \\
\hline
\end{tabular}

Comparison of height $(160.0 \pm 17.6 \& 156.0 \pm 12.8)$ weight $(57.4 \pm 9.9$ $\& 57.3 \pm 8.8)$ and $\mathrm{BMI}(M=22.8 \pm 5.3 \& 23.7 \pm 2.5)$ values of male group using independent-samples t-test showed no statistically significant difference with respective $\mathrm{p}$ values, $\mathrm{p}=0.065, \mathrm{p}=0.980$ and $\mathrm{p}=0.154$ (Figure 1). The comparison of the female groups the mean weight, height and BMI values showed a statistically significant difference with respective $p$ values; $p=0.05$ for the weight and $p=0.0001$ for the height and BMI (Figure 1).

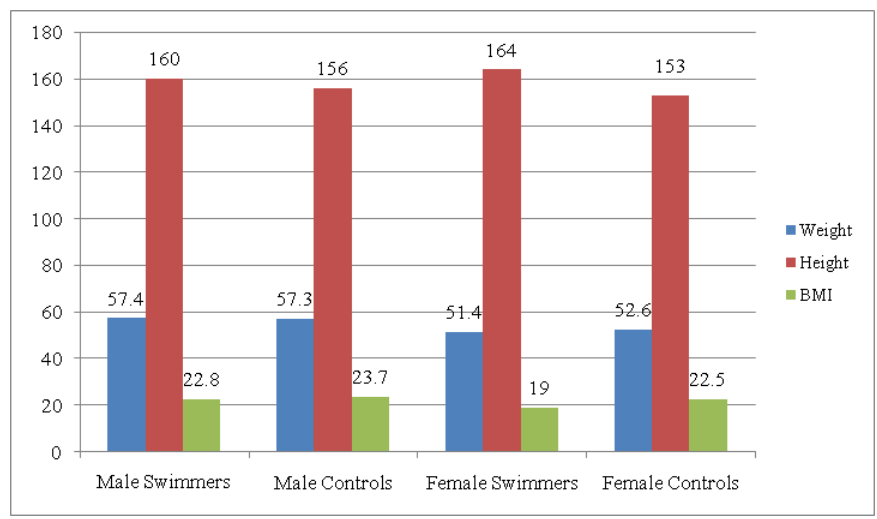

Figure I Comparison of Mean Weight, Height and BMI of the groups.

The mean LBW and FW values of male swimmers were $52.3 \pm 10.4$ and $5.1 \pm 2.1 \mathrm{~kg}$ respectively. Mean LBW of male controls was $49.7 \pm 8.6$ while the mean value for the FW and fat $\%$ were $7.7 \pm 1.9$ and $13.6 \pm 3.9$ in kilogram respectively. For the female swimmers, the mean values of LBW, FW and Fat \% were $42.0 \pm 3.7$ and $9.4 \pm 1.9$ and $18.2 \pm 3.4 \% \mathrm{~kg}$ respectively and for the female controls the mean values of LBW, FW and Fat $\%$ were $39.3 \pm 2.2,13.3 \pm 2.2$ and $25.1 \pm 3.1 \% \mathrm{~kg}$ respectively (Table 2).

Table 2 Comparison of mean values for LBW, FW and Fat \% of the groups $(P \leq 0.05)$

\begin{tabular}{llll}
\hline \multirow{2}{*}{ Group } & \multicolumn{3}{l}{ Mean } \\
\cline { 2 - 4 } & LBW (kg) & FW (kg) & Fat\% \\
\hline Male Swimmers & $52.3 \pm 10.3$ & $5.1 \pm 2.1$ & $9.3 \pm 4.6$ \\
Male Controls & $48.7 \pm 8.6$ & $7.7 \pm 1.9$ & $13.6 \pm 3.9$ \\
Female Swimmers & $42.0 \pm 3.7$ & $9.4 \pm 1.9$ & $18.2 \pm 3.4$ \\
Female Controls & $39.3 \pm 2.2$ & $13.3 \pm 2.2$ & $25.1 \pm 3.1$ \\
\hline
\end{tabular}

Comparison of LBW of male swimmers and controls $(M=52.3 \pm 10.3 \& M=48.7 \pm 8.6)$ using independent sample $t$ test has shown no statistically significant difference with $\mathrm{p}=0.067$. However the comparison of the fat weight of male swimmers non-swimmers $(M=5.1 \pm 2.1 \& M=7.7 \pm 1.9)$ and Fat percentage of the male swimmers and male non swimmers $(M=9.3 \pm 4.6 \& M=13.6 \pm 3.9)$ showed a statistical significance with respective $\mathrm{p}$ values $\mathrm{p}=0.001 \& \mathrm{p}=0.0001$.

A statistically significant difference was observed for the mean LBW (42.0 $\pm 3.7 \& 39.3 \pm 2.2)$, FW (9.4 $\pm 1.9 \& 13.3 \pm 2.2$ ), Fat $\%$ $(\mathrm{M}=18.2 \pm 3.4 \& \mathrm{M}=25.1 \pm 3.1)$ of female swimmers and non-swimmers with same $p$ value $p=0.0001$ (Figure 2 ).

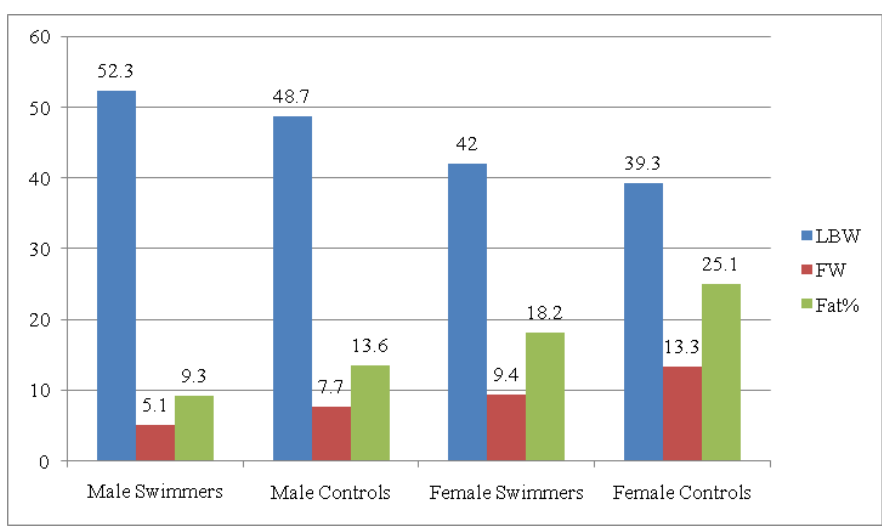

Figure 2 Comparison of Mean values of LBW, FW and Fat $\%$ of the groups.

\section{Discussion}

As observed in the male swimmers and male non swimmers, the mean values of weight were much closer to each other and showed no statistical significance (Table 1). However, in female swimmers and female non swimmers the difference of mean body weight was more than one kilogram. Therefore, female non swimmers in the study were a little heavier than the female swimmers. This difference was statistically significant with $\mathrm{p}$ value equal to 0.05 (Table 1 ). The regular training the development of the muscle mass may be the reason for this observation.

Above result regarding boys is in accordance with a study published by Kumar et al., ${ }^{11}$ where they have described that young athletes show no significant difference in weight with age and sex matched athlete groups. Different authors state that increases in body mass had been observed internationally in children since the $1980 \mathrm{~s}^{12}$ but in contrary, 
this study did not show higher body mass in swimmers compared to non swimmers. The study published by Kondrič et al., ${ }^{13}$ showed that the body mass (BM) allows swimmers to achieve a higher moment of force (MF) but we did not observe this in our swimmers (Table 1) as the mean difference weight is relatively small though some authors have observed a decrease in weight along with body fat associated with increased performance in swimming, that is lighter swimmers have a performance advantage. ${ }^{14}$

There was no significant difference in heights of the male swimmers (MS) and controls $(p=0.065)$ (Table 1) though the difference in heights of female swimmers and female controls was statistically significant (Table 1) $(\mathrm{p}=0.0001)$. Those results were partially in accordance with the results by Geladas, where he described that the height is a significant predictor of sprinters in swimming. The author further goes on to state that the degree of association in girls was markedly lower than in boys. ${ }^{15}$

Another study showed that the pubescent swimmer boys were $8 \%$ taller than non-athletes and the pubescent swimmer girls were about 5\% taller than non-athletes. ${ }^{8}$ Some other studies also show that both swimmer boys and girls tend to be taller than non-athletes of the respective gender and when they continue to involve in the sport they become taller and perform better. ${ }^{8,13,16}$ Results of the present study show that the teen age boys are 3\% and girls are $7 \%$ taller than the non athletes. Some authors further suggested that height is the best predictor of performances in swimming. ${ }^{17,18}$ However, results of our studies do not fully agree with finding of above authors as male group doesn't show a statistical significance in height comparison.

According to the WHO reference for BMI for South East Asian countries the BMI measurements in the male swimmers, male nonswimmers and female non swimmers in our study are in the normal range (Table 1) while value for the female swimmers, fallen to a lesser value $\left(19.03 \mathrm{~kg} / \mathrm{m}^{2}\right)$. Comparison of BMI of the males $(22.81$ \& 23.70 in $\mathrm{kg} / \mathrm{m}^{2}$ respectively in male swimmers and non-swimmers - Table 1$)$ shows no statistically significant difference $(p=0.15)$. This is due to the fact that the body weight and height of the males didn't show a statistically significant difference. Among the girls there is a significant difference in their BMI with a higher probability value, $\mathrm{p}=0.0001$. As both body weight and body height were significantly different we observed a significant difference in BMI between female swimmers and female non swimmers (Table 1)

A significantly higher BMI observed in female non swimmers may have been due to lack of physical activity and similar observations have been reported by Russle et al. \& Kumar et al. ${ }^{11,19}$ The results support the idea that swimming could be used as a means to reduce obesity which is becoming a burning problem all over the world. ${ }^{11,20,21}$

The male subjects have a higher lean body weight (LBW) than female subjects as expected. However, comparison of the mean LBW of male swimmers (MS) and male non-swimmers (MNS) were not statistically significant $(\mathrm{p}=0.066)$. But in case of females, the difference of LBW is significant at the $p=0.0001$. Lean body weight of female swimmers was higher than the controls, even though female controls were significantly heavier than the swimmers. Thus we observed that the fat deposition of female swimmers is lower than non-athletic female group (Table 2).

The reason for this observation may be due to the structured regular training those swimmers under goes. Results of a study conducted by Lieber et al., ${ }^{22}$ to compare athletic and non-athletic young individuals also support this finding. It had shown that a significant difference was found among groups of swimmers and non-athletic group $(\mathrm{p}<0.01)$ for changes in percent body fat, and changes in lean and fat weight over the training period and concludes that the sports and an organized training can directly influence physical anthropometric parameters such as LBW and body fat. ${ }^{22}$

Based on the findings of Zuniga et al., ${ }^{23}$ that is; the swimming performance for girls may be improved through training programs designed to reduce body fatness and decreased weight and body fat are associated with increased performance and the lighter swimmers have a performance advantage. ${ }^{1,5,14,24}$ It can be concluded that increased lean body mass may lead to improved performance and better to keep swimmers with higher lean body mass (less body fat) due to same reason (The sample was national level swimmers in Sri Lanka).

The males have shown low fat weight than females as reported by many authors ${ }^{11,21}$ a fact known widely. In the present study the male swimmers have shown the lowest fat weight which was statistically significant $(\mathrm{p}=0.0001)$ compared to male controls (Table 2$)$. The fat weight comparison of female swimmers and controls also shows a statistical significance with a $\mathrm{p}$ value of 0.0001 . Female swimmers also had shown lower fat weight than the controls. Therefore, it is clear that the swimmers had lower fat weight than non-swimmers. Previous research findings showed that the deposition of fat differs in each gender ${ }^{21}$ and individuals with lower physical fitness were fatter. ${ }^{12}$ Swimming may improve physical fitness and therefore fat weight was less in swimmers and further the training could result lower fat percentage than non-athlete children. It reveals that swimming may influence body fat levels. ${ }^{7,8,11,19,22}$ Therefore, it is clear those findings of the present study are in agreement with the published data.

In contrary to this view, several authors stated say that elite swimmers may get an advantage of having higher body fat levels considering the energy expenditure and some swimming mechanics. ${ }^{5,25,26}$ However, even though we used national level Sri Lankan swimmers in our study the finding was not in agreement with the above hypothesis.

The fat weight of the female swimmers was higher than the male controls (Table 2) and this may be due to the effects of teen age of the respective genders as fat percentage is generally higher in girls than the boys in teen age as a whole. A conclusion could be made that swimming could keep fat weight at a lower level in swimmers in either gender. It further emphasizes that swimming could be used as an exercise component of reducing body fat or body composition as suggested by White et al., ${ }^{10}$ a few decades ago.

Percentage wise also the males have shown lower values while females have shown higher values for body fat (Table 2). Female non swimmers had highest fat percentage followed by female swimmers. The male swimmers had the lowest fat percentage (9.26\%) among the groups, and this was significant compared to the male control group (13.64\%). Though the male control had a higher value it could be considered a desirable value and the reason being that these are teenage subjects. Comparison between female groups also showed a significant difference in fat percentages (18.2 in swimmers and 25.1 in non swimmers) with a $p$ value of 0.0001 . These results show that the swimming may directly influence fat percentage of the body. These observations are in agreement with the findings that fat percentage of swimmers was less than non-swimmers and girls have got higher fat percentages compared to boys. The training could result lower fat percentage than non-athlete children revealing that swimming can influence on body fat percentage levels as it influence on fat mass. ${ }^{7,8,11,19,22}$ 


\section{Conclusion}

According to the results and discussion above we can conclude that the fat weight and fat percentage is less in swimmers than non swimmers and fat deposition of female swimmers is lower than nonathletic female group. The reason for this observation may be due to the structured regular training those swimmers under goes.

The changes in percent body fat, lean body weight and fat weight over the training period and conclude that the sports and an organized training can directly influence physical anthropometric parameters such as LBW and body fat. As the sample is teenagers a contribution of the effect of hormones may possible and further research are needed to identify those effects.

Therefore, it is clear that the swimmers had lower fat weight than non-swimmers. It further emphasizes that swimming could be used as an exercise component of reducing body fat or body composition as suggested by White et al., ${ }^{10}$ a few decades ago.

The height weight and the BMI may not have much effect in the swimming perspective of the males but there were significant relationship in those parameters in females.

\section{Acknowledgements}

We are thankful to the participant in this study, the swim coaches, the staff of the Allied Health Sciences Unit, Faculty of Medicine and the staff of the laboratories and department of physiology, Faculty of Medicine, University of Peradeniya.

\section{Conflict of interest}

The author declares no conflict of interest.

\section{References}

1. Santos SSD, Riehle H. Relationship among Anthropometric Characteristics, Stroke Frequency and Stroke Length in Brazilian Elite Swimmers. ISBS- Conference, Germany; 1998.

2. Herbet HR. The Encyclopaedia of Occupational Health and Safety. $J$ Occupational Medicine. 1984;26(4):253-254.

3. Cogill B. Anthoropometric Indicators Measuring Guide. USA: Food \& Nutrition technical Assistance Project., Academy for Educational Development; 2001.

4. Stamm R, Stamm M. Anthropometric factor in the assessment of young females. The Mankind Quarterly, University of Estonia; 2004.

5. Knechtle B, Baumann B, Knechtle A, et al. A Comparison Between Iron Man Triathletes and Ultra Swimmers. Journal of Human Kinetics. 2004;24:57-64.

6. Coelho J, Cruz AM, Marinho DA, et al. Preliminary Attempt to Develop a Path-Flow Analysis Model for Swimming Performance in Children. The open sport science journal. 2010;3:7-9.

7. Emslander HC, Sinaki M, Muhs JM, et al. Bone mass and muscle strength in female college athletes [runners and swimmers]. Mayo Clin Proc. 1998;73(12):1151-1160.

8. Schneider P, Meyer F. Anthropometric and Muscle Strength Evaluation in Prepubescent and Pubescent Swimmer Boys and Girls. Rev Bras Med Esporte. 2005:11(4).

9. Cox GR, Broad EM, Riley MD, et al. Body Mass Changes and Voluntary Fluid Intake of Elite Level Water Polo Players and Swimmers. J Sci Med Sport. 2002;5(3):183-193.
10. White GM, Young RJ. Effect of Twelve Weeks Exercise Program on Cardio-Respiratory and Body Composition Variables in Young, None Obese Middle Aged Females. Br J Sports Med. 1978;12(1):27-32.

11. Kumar S, Sharma VK, Vinayathan A. Comparison of Effect of Regular Unstructured Physical Training and Athletic Level Training on Body Composition and Cardio Respiratory Fitness in Adolescents. J Clin Diagn Res. 2013;7(9):1878-1882.

12. Albon HM, Hamlin MJ, Ross JJ. Secular trends and distributional changes in health and fitness performance variables of 10-14-year-old children in New Zealand between 1991 and 2003. Br J Sports Med. 2008;44(4):263-269.

13. Kondrič M, Uljević O, Gabrilo G, et al. General Anthropometric and Specific Physical Fitness Profile of High-Level Junior Water Polo Players. J Hum Kinet. 2012;32:157-165.

14. Reel JJ, Gill DL. Slim Enough to Swim? Weight Pressures for Competitive Swimmers and Coaching Implications. The Sport Journal. 2001.

15. Geladas ND, Nassis GP, Pavlicevic S. Somatic and physical traits affecting sprint swimming performance in young swimmers. Int J Sports Med. 2005;26(2):139-144.

16. Young WB, Newton RU, Doyle TL, et al. Physiological and Anthropometric Characteristics of Starters and Non-Starters and Playing Positions in Elite Australian Rules Football: A Case Study. J Sci Med Sport. 2005;8(3):333-345.

17. Zampagni ML, Casino D, Benelli P, et al. Anthropometric and strength variables to predict freestyle performance times in elite master swimmers. J Strength Cond Res. 2008;22(4):1298-1307.

18. Jagomägi G, Jürimäe T. The influence of anthropometrical and flexibility parameters on the results of breaststroke swimming. Anthropol Anz. 2005;63(2):213-219.

19. Pate RR, Davis MG, Robinson TN, et al. Promoting Physical Activity in Children and Youth; A Leadership Role for Schools: A Scientific Statement From the American Heart Association Council on Nutrition, Physical Activity, and Metabolism (Physical Activity Committee) in Collaboration With the Councils on Cardiovascular Disease in the Young and Cardiovascular Nursing. Circulation. 2006;114:1214-1224.

20. Chatwal J, Verma M, Riar SK. Obesity among pre-adolescent and adolescents of a developing country (India). Asia Pac J Clin Nutr. 2004;13(3):231-235.

21. Abu Hanifah R, Mohamed MN, Jaafar Z, et al. The Correlates of Body Composition with Heart Rate Recovery after Step Test: An Exploratory Study of Malaysian Adolescents. PLoS One. 2013;8(12):e82893.

22. Lieber DC, Lieber RL, Adams WC. Effects of run-training and swimtraining at similar absolute intensities on treadmill VO2max. Med Sci Sports Exerc. 1989;21(6):655-661.

23. Zuniga J, Housh TJ, Mielke $M$, et al. Gender comparisons of anthropometric characteristics of young sprint swimmers. J Strength Cond Res. 2011;25(1):103-108.

24. Tanda G, Knechtle B. Marathon performance in relation to body fat percentage and training indices in recreational male runners. Open Access J Sports Med. 2013;4:141-149.

25. Burke L. Swimmers Body Fat Mystery. Australia: Australian Institute of Sports; 1997.

26. Flynn MG, Costill DL, Kirwan JP, et al. Fat Storage in Athletes: Metabolic and Hormonal Responses to Swimming and Running. Int J Sports Med. 1990;11(6):433-440. 